\title{
Evaluation of an Embodied Virtual Reality Device in Upper Limb Rehabilitation Post-Stroke
}

\author{
A. El-Hilly, R. Harris, N. Gillion, C. Cole, A. Dimaano, J. Johnson, E. Lawrence, Y.Y.K. Kee, \\ Department of Stroke \& Elderly Care, Croydon University Hospital
}

Croydon University Hospital, Stroke Department, Care of the Elderly, Croydon, United Kingdom

\section{Introduction}

There is increased evidence that intensity in therapy is important in improving outcomes after stroke. Current United Kingdom recommendations state that patients should receive at least 45 minutes of each required therapy, 5 days a week. Virtual reality gaming platforms is an innovative method of improving engagement and intensity of rehabilitation.

This is a small feasibility study to explore the use and efficacy of an immersive virtual reality platform for intensive upper limb rehabilitation on an acute stroke unit.

\section{Methods and Patients}

\section{Results}

- All patients saw an improvement in their scores (See Tables).

- The number of repetitions achieved by end of week 4 increased between $50-92 \%$ for all 3 patients.

- 2 patients showed a clinically important difference (6.3pts) from the CAHAl-7.

-Therapists described ease of use of the platform.

- Patients reported positive effects on mental health and enjoyed the ability to monitor their progress easily.

"I found that the sessions helped with my mental ability during therapy"

"I appreciate the serious side of this technology but there is a novelty factors which could be quite attractive to some users"

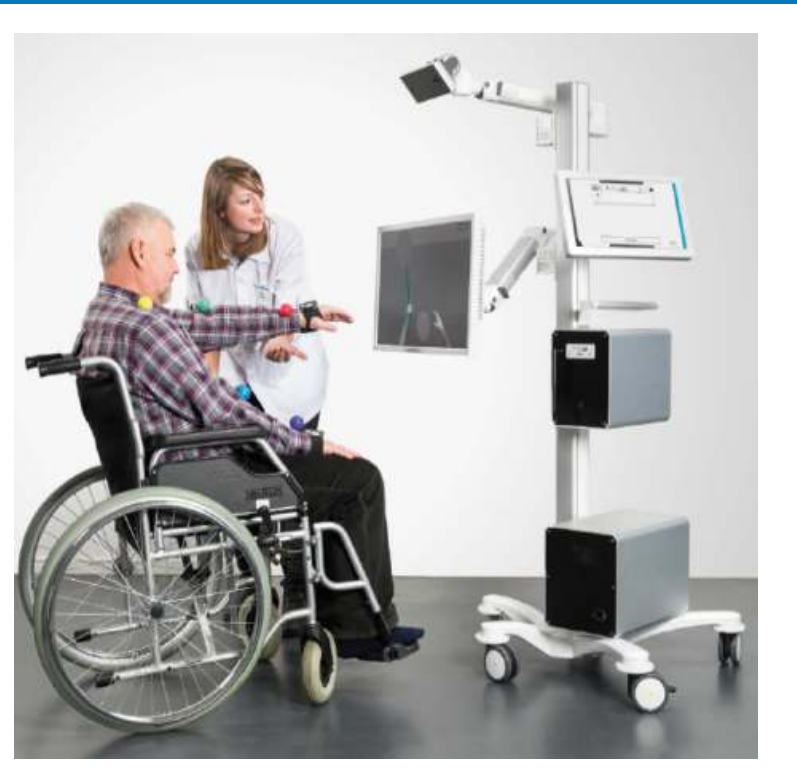

Figure 1 : MindMotion PRO

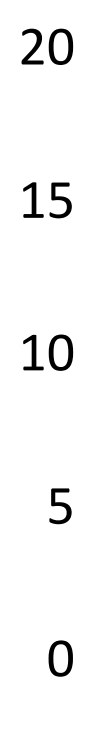

20

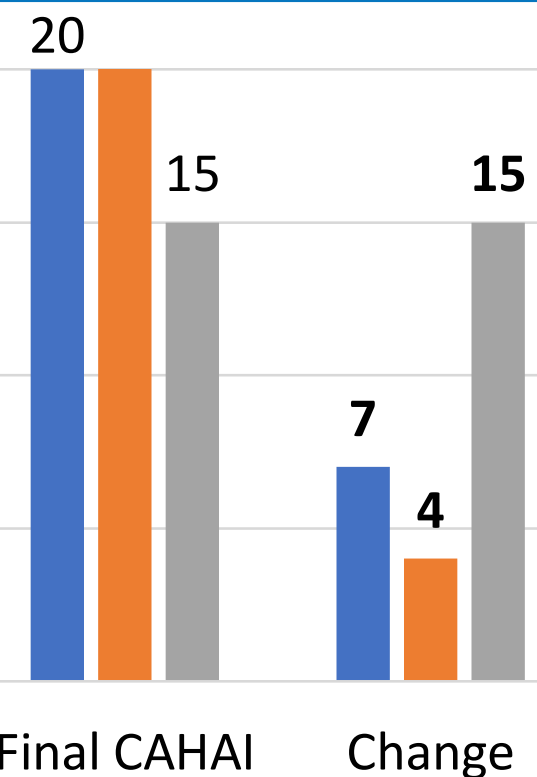

Initial CAHAI Final CAHAI Change

Table 1 : CAHAI Scores

Initial number of repetition

Final number of reps

Change

Sessions $\quad 7^{911}$

Patient $1 \quad$ Patient $2 \quad$ Patient 3

$\begin{array}{lllll}0 & 20 & 40 & 60 & 80\end{array}$

Table 2: Number of Repetitions and Sessions

\section{Discussion}

- Despite initial skepticism from patients and therapists about the use of virtual reality in stroke rehabilitation, there was good response from both staff and patients.

- Both groups liked the ability to be able to see the progress made each session.

- Both groups acknowledged that this therapy is an useful adjunct to conventional therapy.

\section{Conclusion}

The MindMotion PRO shows promise in providing engaging, enjoyable and effective rehabilitation for stroke patients whilst helping to meet therapy targets. It has the potential of improving intensity of therapy especially on weekends. Further work is needed to establish cost-efficacy and to explore long term benefits for various patient cohorts.

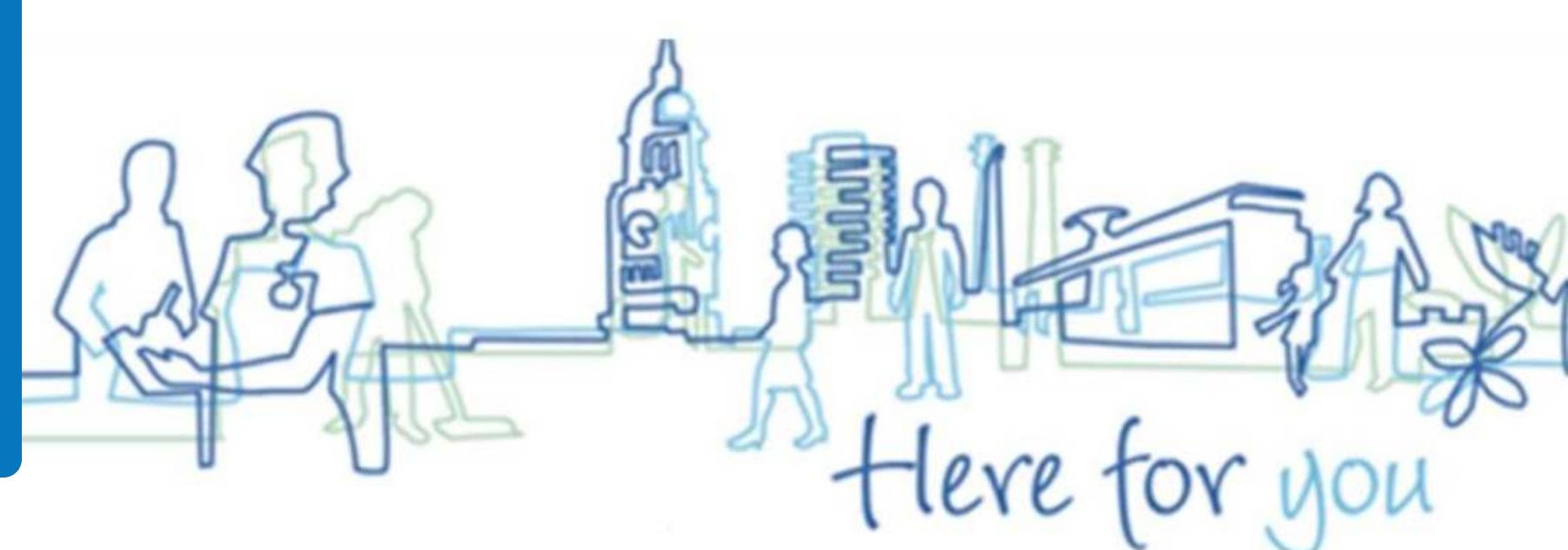

